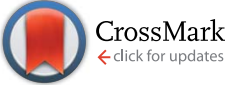

Cite this: RSC Adv., 2017, 7, 10631

\title{
Morphology-controlled synthesis and electron field emission properties of ZnSe nanowalls
}

\author{
Pei Xie, ${ }^{\text {ab }}$ Shaolin Xue, ${ }^{\text {b }}$ Youya Wang, ${ }^{\mathrm{b}}$ Zhiyong Gao, ${ }^{\mathrm{b}}$ Hange Feng, ${ }^{\mathrm{b}}$ Lingwei Li, ${ }^{\mathrm{b}}$ \\ Dajun Wu, ${ }^{\mathrm{C}}$ Lianwei Wang ${ }^{\mathrm{C}}$ and Paul K. Chu ${ }^{\mathrm{d}}$
}

ZnSe nanowalls are prepared on Zn substrates hydrothermally at $180{ }^{\circ} \mathrm{C}$ for $12 \mathrm{~h}$. The structure, morphology, and chemical composition of the ZnSe nanowalls are determined by X-ray diffraction (XRD), scanning electron microscopy (SEM), transmission electron microscopy (TEM), energy-dispersive $X$-ray analysis (EDS), and Raman scattering. The concentration of hydrazine hydrate $\left(\mathrm{N}_{2} \mathrm{H}_{4} \cdot \mathrm{H}_{2} \mathrm{O}\right)$ plays an important role in the formation and morphology of the ZnSe nanowalls and XRD reveals that all the ZnSe nanocrystals have a cubic zinc blende structure. The ZnSe nanowalls with an optimal morphology deliver a good field electron emission performance with a turn-on field of $1.51 \mathrm{~V}^{-1}$ and enhancement factor of 4797. The formation mechanism is discussed. The simple and controlled preparation procedures and good properties of the ZnSe nanowalls bode well for application in electronics and optoelectronics.

Received 30th November 2016 Accepted 30th January 2017

DOI: 10.1039/c6ra27541a

rsc.li/rsc-advances diodes, ${ }^{22}$ blue color light emitting devices, ${ }^{23}$ gas sensors, ${ }^{24}$ photo detectors, ${ }^{25}$ and catalyst supports. ${ }^{26}$ Hao et al. ${ }^{27}$ synthesized ZnSe spheres with good photoelectrochemical properties hydrothermally and Zeng et al. ${ }^{28}$ fabricated ZnSe nanocones and nanobelts on $\mathrm{Zn}$ foils and the materials delivered high field emission performance and had good optical characteristics. Zou et al. ${ }^{29}$ found that the microstructure had a significant effect on the photoluminescence properties of ZnSe nanoribbons and Zannier et al. ${ }^{30}$ used the vapor-solid-solid (VSS) method to fabricate ZnSe nanowires with a controllable diameter. Morphology-controlled growth of 2D ZnSe nanostructures has been demonstrated and their photovoltaic properties, ${ }^{31}$ optical properties, ${ }^{32}$ and photocatalytic properties $^{33}$ have been investigated.

In this work, 2D ZnSe nanowalls with different morphologies are fabricated on a large scale by a hydrothermal method at $180{ }^{\circ} \mathrm{C}$ and the field emission properties are investigated. In the process, the morphology of the $\mathrm{ZnSe}$ nanowalls prepared on $\mathrm{Zn}$ substrates can be easily controlled by adjusting the concentration of $\mathrm{N}_{2} \mathrm{H}_{4} \cdot \mathrm{H}_{2} \mathrm{O}$ so that $\mathrm{ZnSe}$ nanowalls with the optimal field emission performance can be obtained. The ZnSe nanomaterials are promising in applications such as field emission displays.

\section{Experimental section}

201620, China

${ }^{b}$ College of Science, Donghua University, Shanghai 201620, China.E-mail: peipeixie@ 163.com; slxue@dhu.edu.cn; 15026571630@163.com; gaozhiyong0829@163.com; 17721486315@163.com; li.lingwei.pink@163.com; djwu@ee.ecnu.edu.cn; lwwang@ ee.ecnu.edu.cn; paul.chu@cityu.edu.hk; Fax: +862167792089

${ }^{c}$ Key Laboratory of Polar Materials and Devices, Ministry of Education, and Department of Electronic Engineering, East China Normal University, 500 Dongchuan Road, Shanghai 200241, China

${ }^{d}$ Department of Physics and Material Science, City University of Hong Kong, Tat Chee Avenue, Kowloon, Hong Kong, China

\subsection{Materials}

All the reagents used in this work were analytical grade and used as-received without any purification, including the zinc substrates (Zn) ( $\geq 99.0 \%)$ (Sinopharm Chemical Reagent Ltd., China), sodium hydroxide $(\mathrm{NaOH})(\geq 97.0 \%)$ (Pinghu Chemical Reagent Ltd., China), Se powder (Se) ( $\geq 99.7 \%$ ) (Sinopharm Chemical Reagent Ltd., China), hydrazine hydrate $\left(\mathrm{N}_{2} \mathrm{H}_{4} \cdot \mathrm{H}_{2} \mathrm{O}\right.$, 
85\%) (Sinopharm Chemical Reagent Ltd., China), acetone $\left(\mathrm{CH}_{3} \mathrm{COCH}_{3}\right)(\geq 99.7 \%)$ (Beijing Chemical Reagent Ltd., China), ethanol $\left(\mathrm{C}_{2} \mathrm{H}_{5} \mathrm{OH}\right)(\geq 99.7 \%)$ (Beijing Chemical Reagent Ltd., China). The aqueous solutions were prepared with deionized water.

\subsection{Synthesis of the ZnSe nanowalls}

The typical synthesis is described as follows. $0.5 \mathrm{~g}$ of Se powder, $65 \mathrm{ml}$ of $\mathrm{NaOH}(0.24 \mathrm{~mol})$, and $12 \mathrm{ml}$ of $\mathrm{N}_{2} \mathrm{H}_{4} \cdot 2 \mathrm{H}_{2} \mathrm{O}$ were added to a $100 \mathrm{ml}$ beaker under magnetic stirring. After 30 minutes, the Se powder was dissolved to form the solution. The $\mathrm{Zn}$ substrate with width of $1 \mathrm{~cm}$, length of $1 \mathrm{~cm}$ and thickness of 0.3 $\mathrm{mm}$ was utilized also as a reactant. The $\mathrm{Zn}$ substrate was ultrasonically cleaned in acetone for $10 \mathrm{~min}$ and rinsed with ethanol and deionized water several times was immersed in the solution. The solution with the $\mathrm{Zn}$ substrate was put in a $100 \mathrm{ml}$ Teflon-lined stainless steel autoclave and heated to $180{ }^{\circ} \mathrm{C}$ in an air-flow electric oven for $12 \mathrm{~h}$. Afterwards, the autoclave cooled naturally to room temperature and the $\mathrm{Zn}$ substrate was taken from the solution. Finally, the substrate deposited with ZnSe nanosheets was washed with deionized water and ethanol several times sequentially and dried at room temperature under vacuum before use. ZnSe samples with different morphologies were prepared by changing the concentration of $\mathrm{N}_{2} \mathrm{H}_{4} \cdot 2 \mathrm{H}_{2} \mathrm{O}$ in the solution. The samples obtained using $0,5,12$, and $14 \mathrm{ml}$ of $\mathrm{N}_{2} \mathrm{H}_{4} \cdot 2 \mathrm{H}_{2} \mathrm{O}$ were termed as Samples 1-4 hereafter for simplicity.

\subsection{Characterization}

The crystal structure and phase composition of the ZnSe nanowalls were determined by X-ray diffraction (XRD; RigakuDmax 2550, Japan) with $\mathrm{Cu} \mathrm{K}_{\alpha}$ radiation $(\lambda=1.5406 \AA \hat{A})$ at 40 $\mathrm{kV}$ and $200 \mathrm{~mA}$ in a $2 \theta$ range of $20-70^{\circ}$. The composition of the sample was determined by X-ray energy dispersive spectroscope (EDS; Oxford IE $300 \mathrm{X}$ ) and the surface morphology was examined by scanning electron microscopy (SEM; JEOL JSM-5600LV), transmission electron microscopy (TEM; Hitachi H-600), and high-resolution transmission electron microscopy (HR-TEM; JEOL JEM-2100). The crystallinity of the ZnSe nanowalls was evaluated by Raman scattering (JY LabRam-1B).

\subsection{Field emission measurements}

The as-prepared sample pasted on polished Al substrate was used as the cathode and the ITO glass was used as the anode. In the measurements, the distance between electrodes was kept $\sim 500 \mu \mathrm{m}$ to form a diode structure in the field emission system. Field emission properties of the samples were measured in a ball-type vacuum chamber under a pressure of $\sim 5 \times 10^{-4} \mathrm{~Pa}$ and the voltage was swept from $0 \mathrm{~V}$ up to $3400 \mathrm{~V}$ with an interval of $50 \mathrm{~V}$ to measure the corresponding field emission current.

\section{Results and discussion}

The XRD spectra of the as-synthesized samples are depicted in Fig. 1 and can be indexed to the cubic zinc blende ZnSe with $a=$ $5.669 \AA$ of the JCPDS file no.80-2346. The diffraction peaks of the
ZnSe nanowalls are assigned to the (1 111 ), (2 $\left.\begin{array}{lll}2 & 0\end{array}\right),\left(\begin{array}{lll}2 & 2 & 0\end{array}\right),\left(\begin{array}{lll}3 & 1 & 1\end{array}\right),\left(\begin{array}{l}2 \\ 2\end{array}\right.$ 22 ) and ( $\left.\begin{array}{lll}4 & 0 & 0\end{array}\right)$ planes and no characteristic peaks of other crystalline forms are detected by XRD. The composition of the ZnSe nanosheets (Sample 3) determined by EDS indicates that the sample is composed of $\mathrm{Zn}$ and Se with a molar ratio of about $1: 1$.

In order to investigate the influence of the concentration of $\mathrm{N}_{2} \mathrm{H}_{4} \cdot 2 \mathrm{H}_{2} \mathrm{O}$ on the morphology of the ZnSe nanowalls, different experimental procedures are implemented and the products are examined by SEM. Fig. 2(a-d) show the SEM images of $4 \mathrm{ZnSe}$ nanowalls prepared with different concentrations of $\mathrm{N}_{2} \mathrm{H}_{4} \cdot 2 \mathrm{H}_{2} \mathrm{O}$. As shown in Fig. 2(a), several thick and cluttered wall-like microstructures with the thickness of 60-100 nm are formed without $\mathrm{N}_{2} \mathrm{H}_{4} \cdot 2 \mathrm{H}_{2} \mathrm{O}$ and the nanowalls are distributed irregularly. In the presence of $5 \mathrm{ml} \mathrm{N}_{2} \mathrm{H}_{4} \cdot 2 \mathrm{H}_{2} \mathrm{O}$, more uniformly distributed and thinner nanowalls are formed, as shown in Fig. 2(b). Fig. 2(b) also shows that the nanowalls with the thickness of approximately $36 \mathrm{~nm}$ are thinner than those shown in Fig. 2(a). Fig. 2(c) reveals that the nanowalls are made of thin sheets with the thickness of $20 \mathrm{~nm}$ uniformly distributed on the $\mathrm{Zn}$ substrate when the amount of $\mathrm{N}_{2} \mathrm{H}_{4} \cdot 2 \mathrm{H}_{2} \mathrm{O}$ is increased to $12 \mathrm{ml}$. Obviously, the ZnSe nanomaterials shown in Fig. 2(c) have been formed with the thinnest wall compared to Fig. 2(a) and (b). As shown in Fig. 2(d), if the amount of $\mathrm{N}_{2} \mathrm{H}_{4} \cdot 2 \mathrm{H}_{2} \mathrm{O}$ exceeds $12 \mathrm{ml}$, the thin ZnSe nanowalls with the thickness of approximately $27 \mathrm{~nm}$ also uniformly distribute on the $\mathrm{Zn}$ substrate but these nanostructures are curlier than those in Fig. 2(c). In addition, the edges of these nanowalls shown in Fig. 2(c) are sharper and the structures are more symmetrical than those of other samples. According to our experimental results, the surface morphology of the ZnSe nanowalls can be controlled by using different concentrations of $\mathrm{N}_{2} \mathrm{H}_{4} \cdot 2 \mathrm{H}_{2} \mathrm{O}$ and the nanostructures with thin and no curly ZnSe nanowalls expected to have the best field emission properties can be produced.

In addition, the influence of the different reaction time on the morphology of ZnSe nanowalls (Sample 3) were carried out with other conditions keeping constant. The SEM images obtained at different reaction time are presented in Fig. 3(a-d). When reaction time was performed for $2 \mathrm{~h}$, random and

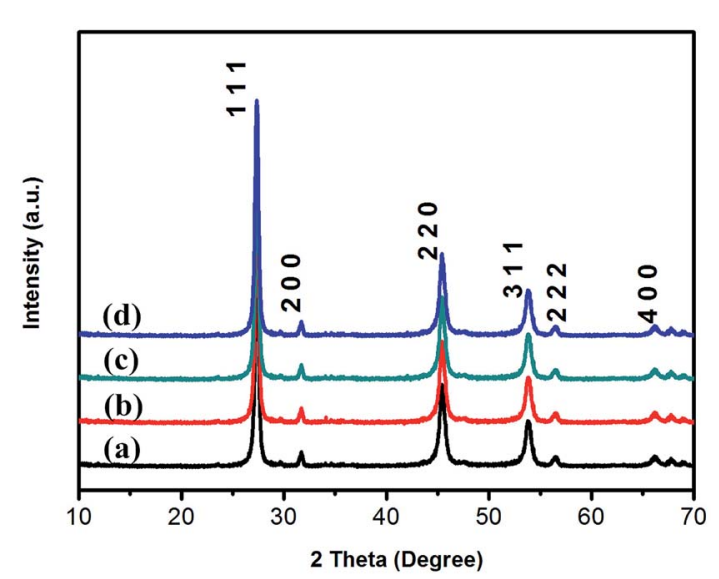

Fig. 1 XRD patterns of the ZnSe nanowalls synthetized at $180{ }^{\circ} \mathrm{C}$ for $12 \mathrm{~h}$ with different concentrations of $\mathrm{N}_{2} \mathrm{H}_{4} \cdot 2 \mathrm{H}_{2} \mathrm{O}$ : (a) Sample 1, (b) Sample 2, (c) Sample 3, and (d) Sample 4. 

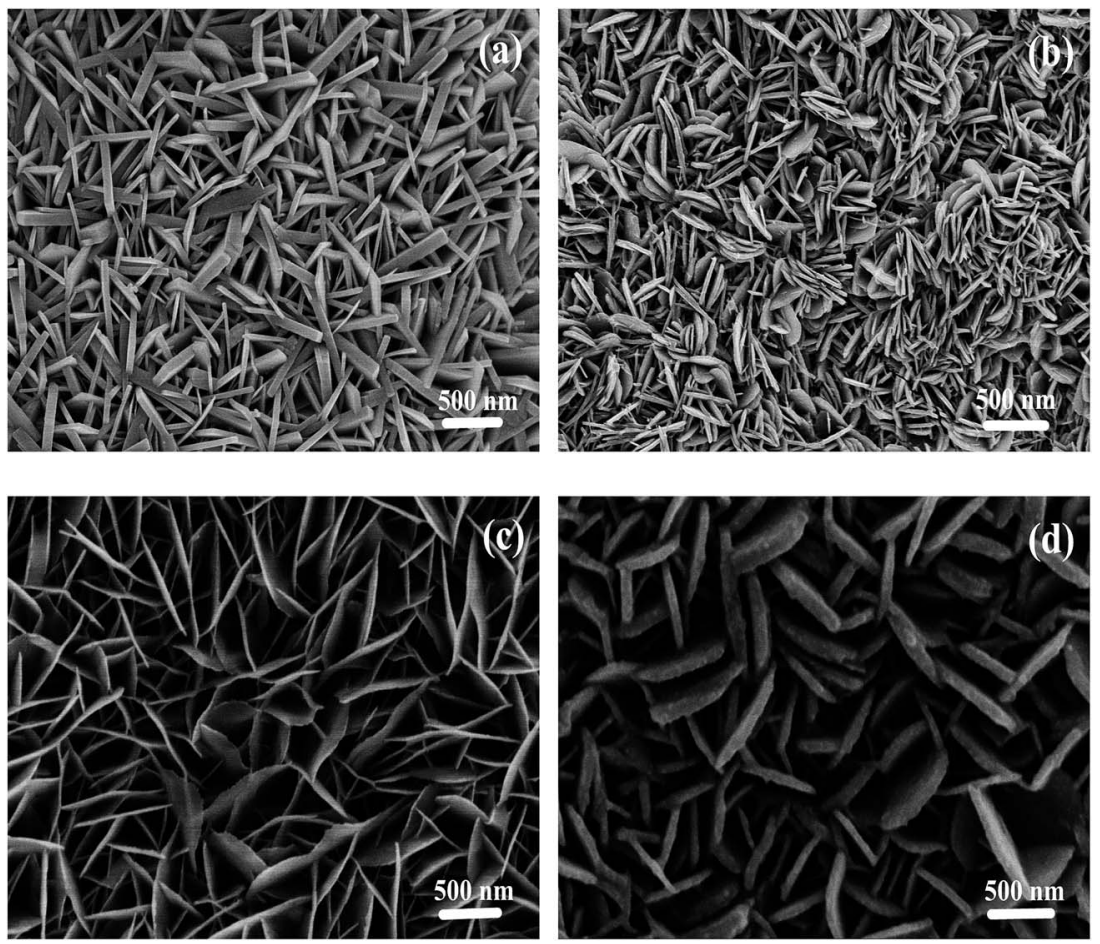

Fig. 2 (a-d) SEM images demonstrating the growth of the ZnSe nanowalls at $180{ }^{\circ} \mathrm{C}$ for $12 \mathrm{~h}$ with $\mathrm{N}_{2} \mathrm{H}_{4} \cdot 2 \mathrm{H}_{2} \mathrm{O}(0,5,12$, and $14 \mathrm{ml}$, respectively).

aggregated ZnSe spherical particles were observed, as shown in Fig. 3(a). When the reaction time was continued for $6 \mathrm{~h}$, underdeveloped sphere-like structures evolve into sheet-like structures with small size completely covered the substrate, as shown in Fig. 3(b). As can be seen from Fig. 3(c), when the hydrothermal reaction time was prolonged to $10 \mathrm{~h}$, a large quantity of rough ZnSe nanowalls with large size gradually grow, but there are still some aggregates. Along with the prolonged reaction time $(12 \mathrm{~h})$, large-scale regular and uniform nanowalls are formed, as shown in Fig. 2(c).

A formation mechanism of ZnSe nanowalls (Sample 3) based on the different reaction time was proposed. Initially, nuclei are formed in the solution and then self-assembled to form small sheet-like structures. As reaction proceeded further, the presence of these assembled nanosheets further develop to form nanowalls with large size. The evolution of the ZnSe nanowalls morphology can be explained by the involvement of Ostwald ripening process. The spontaneously Ostwald ripening in the growth process will decrease the surface free energy of the nanocrystals through increasing the size of nanocrystals, resulting in further growth of superior sheet-like architectures. The results suggest that the generation of nanocrystals with small size is crucial for the formation of nanowalls. Therefore, by controlling the hydrothermal reaction time, the uniform ZnSe nanowalls can be obtained.

The phase structure and composition of the obtained ZnSe nanowalls at different reaction time was investigated by XRD. As can be seen from Fig. 3(d), the XRD patterns of the samples obtained after 2, 6, 10 and $12 \mathrm{~h}$ hydrothermal heat treatment show the cubic zinc blende phase. The XRD pattern of the sample prepared by heating for $2 \mathrm{~h}$ shows some peaks from impurities such as Se (JCPDS file no. 47-1516). Besides, the intensity of ZnSe peaks are relatively weak indicating poor crystallinity. As the reaction time was prolonged to $6-10 \mathrm{~h}$, the relative intensity diffraction peaks at $37.7^{\circ}$ and $60^{\circ}$ corresponding to Se become gradually disappeared, whereas the cubic zinc blende phase ZnSe grow rapidly and crystallization becomes better, indicating the formation of $\mathrm{ZnSe}$ from selenium. Finally, when the reaction time reached $12 \mathrm{~h}$, ZnSe nanowalls without impurities were obtained. The results of XRD indicate that the crystallinity of ZnSe nanowalls would be enhanced with extended time.

The morphology and structure of the ZnSe nanowalls (Sample 3) are further investigated by TEM, HRTEM and FFT. Fig. 4(a) and (b) show the low-magnification TEM image of an individual nanowall disclosing that it is flat, smooth, and uniform in width. Fig. 4(c) shows the HR-TEM image of the nanowall in Fig. 4(a). The planes with a spacing of about $0.2 \mathrm{~nm}$ are parallel to the growth direction coinciding with the interplanar spacing of the cubic ZnSe (2 20 ) planes. Fig. 4(d) displays the corresponding FFT pattern of the nanowall confirming that the hierarchical ZnSe structure is single-crystalline.

The ZnSe nanowalls are analyzed by Raman scattering ${ }^{34}$ using the $532 \mathrm{~nm}$ excitation wavelength at room temperature. Fig. 5 shows a dominant peak at $251 \mathrm{~cm}^{-1}$ which can be assigned to longitudinal optical (LO) phonon scattering of the ZnSe crystal. The sharp Raman peak indicates that the ZnSe nanowalls have high crystallinity and a pure phase in good agreement with HRTEM and XRD. Compared to the LO phonon scattering peak at $255 \mathrm{~cm}^{-1}$ of previously reported nanocrystalline $\mathrm{ZnSe}^{35}$ the Raman peak at $250.8 \mathrm{~cm}^{-1}$ shifts to lower frequencies due to the small size and large surface effects. ${ }^{36}$ 

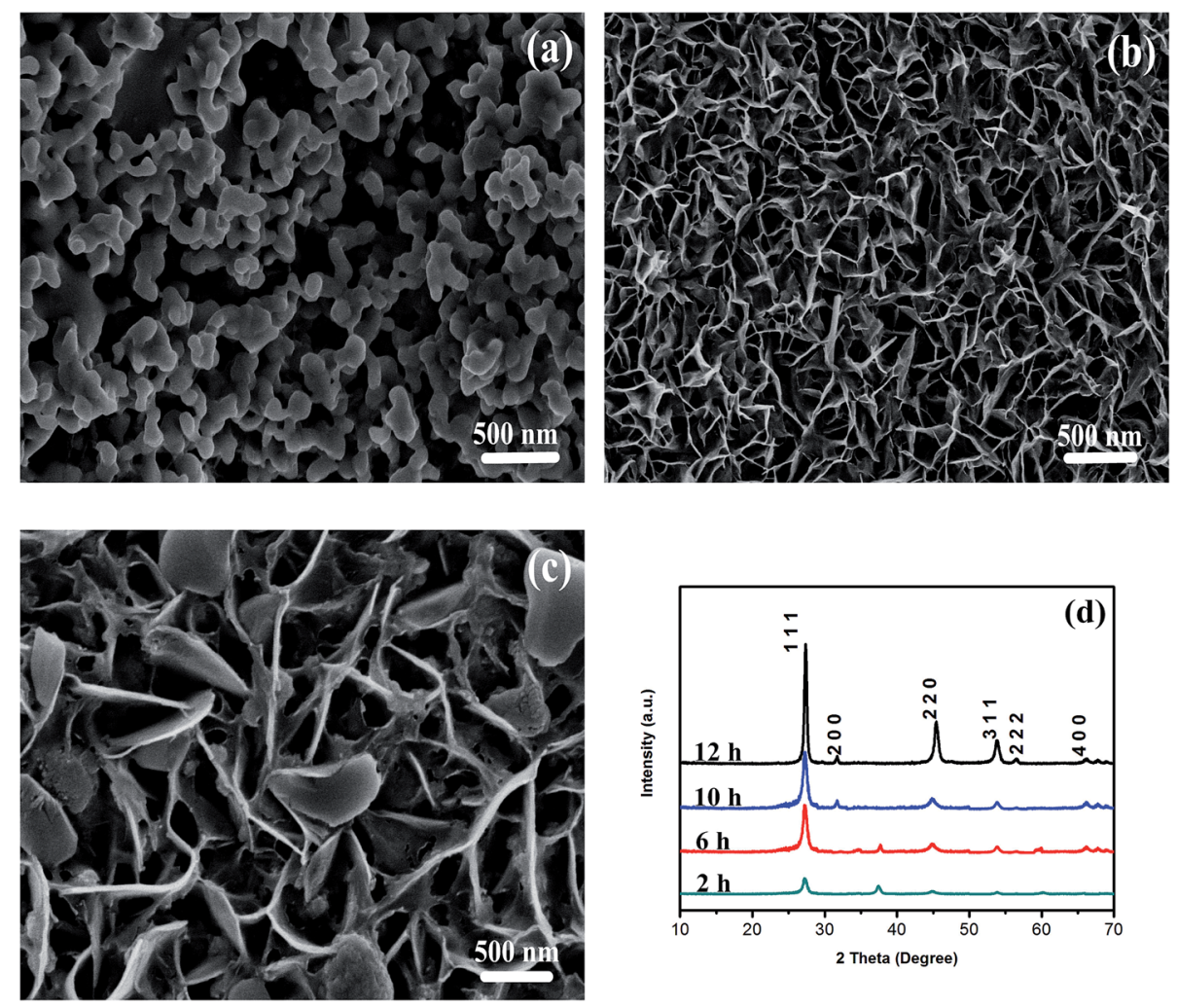

Fig. 3 (a-c) SEM images of the as-prepared ZnSe nanowalls under various reaction time: $2 \mathrm{~h}, 6 \mathrm{~h}, 10 \mathrm{~h}$, respectively. (d) XRD patterns of the asprepared ZnSe nanowalls hydrothermally treated for various time.
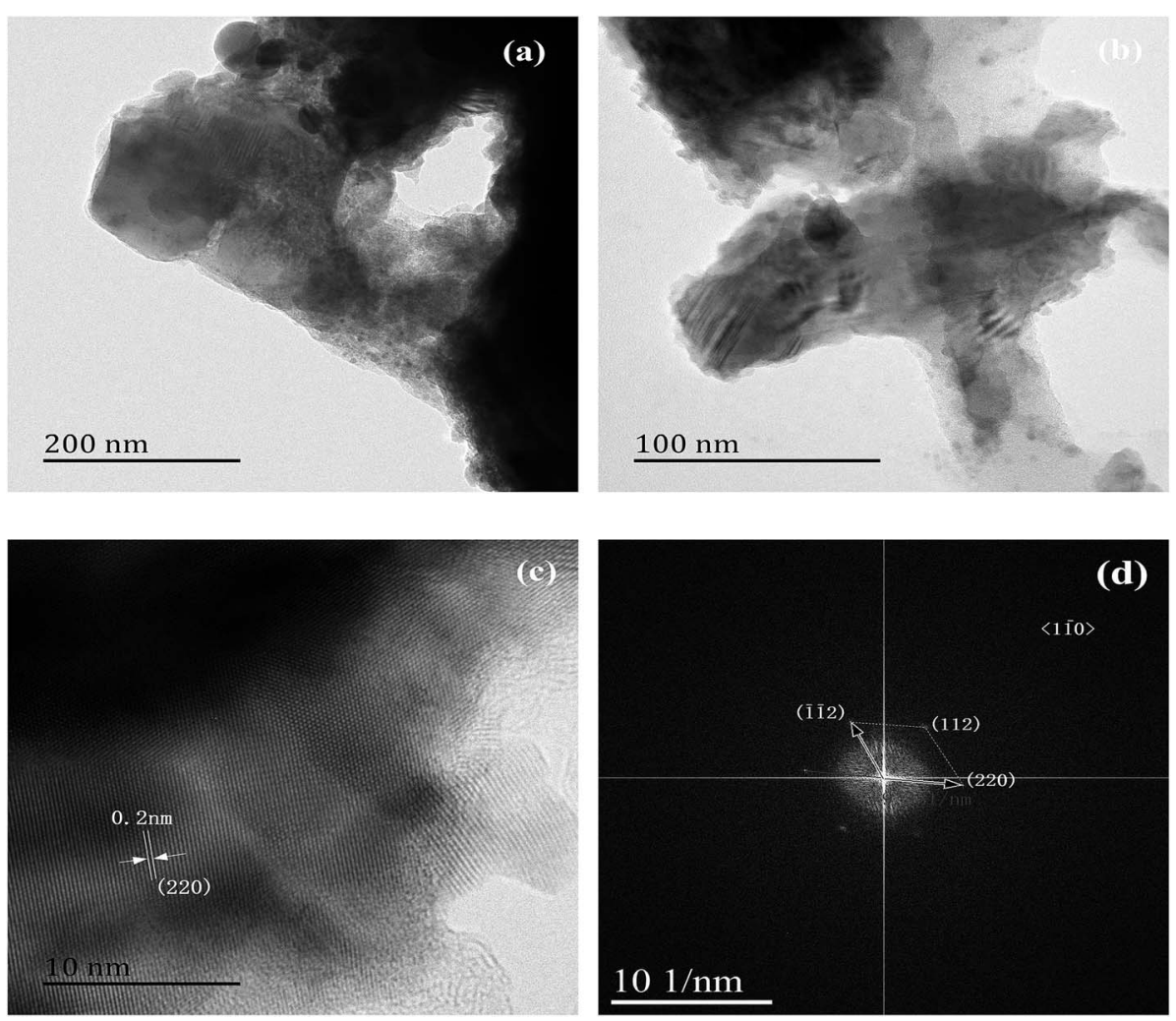

Fig. 4 (a) and (b) TEM images of the nanowall, (c) HR-TEM image of the nanowall, and (d) corresponding FFT pattern (Sample 3). 


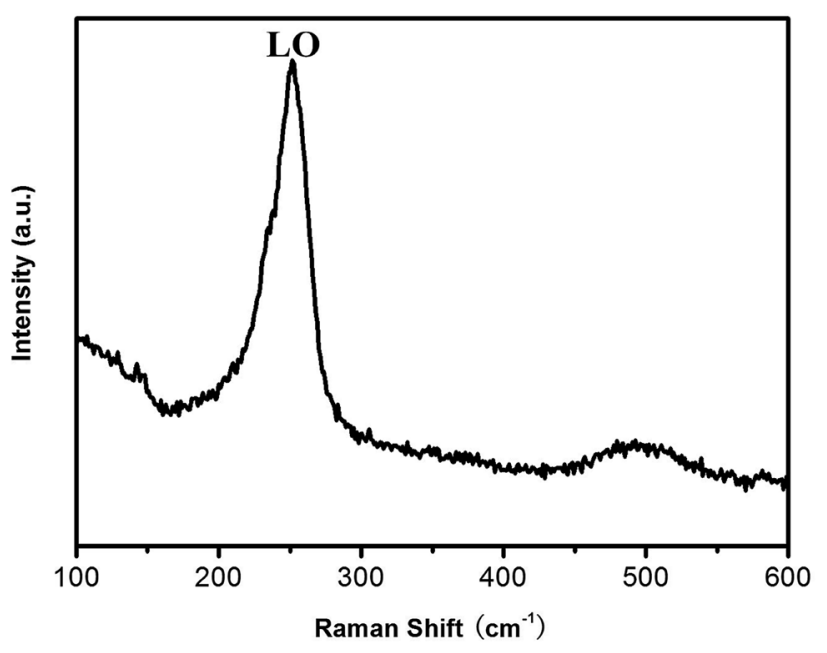

Fig. 5 Raman scattering spectrum obtained from the ordered ZnSe nanowalls (Sample 3).

The chemical reactions are shown in the following:

$$
\begin{gathered}
\mathrm{N}_{2} \mathrm{H}_{4} \cdot 2 \mathrm{H}_{2} \mathrm{O} \leftrightarrows \mathrm{N}_{2} \mathrm{H}_{5}^{+}+\mathrm{OH}^{-} \\
\mathrm{N}_{2} \mathrm{H}_{5}^{+}+2 \mathrm{Se}+5 \mathrm{OH}^{-}=\mathrm{N}_{2}+2 \mathrm{Se}^{2-}+5 \mathrm{H}_{2} \mathrm{O} \\
\mathrm{Zn}^{2+}+2 \mathrm{OH}^{-}=\mathrm{Zn}(\mathrm{OH})_{2} \downarrow \\
\mathrm{Zn}(\mathrm{OH})_{2}+2 \mathrm{OH}^{-}=\left[\mathrm{Zn}(\mathrm{OH})_{2}\right]^{2-}
\end{gathered}
$$

Initially, $\mathrm{N}_{2} \mathrm{H}_{4} \cdot 2 \mathrm{H}_{2} \mathrm{O}$ is decomposed into $\mathrm{N}_{2} \mathrm{H}_{5}{ }^{+}$and $\mathrm{NaOH}$ is added to maintain a stable alkaline environment for the reactions. $\mathrm{N}_{2} \mathrm{H}_{5}{ }^{+}$reacts with Se particles to form $\mathrm{Se}^{2-}$ and $\mathrm{OH}^{-}$protects $\mathrm{Se}^{2-}$ from oxidization. $\mathrm{OH}^{-}$also ensures that $\mathrm{Zn}^{2+}$ does not form $\mathrm{Zn}(\mathrm{OH})_{2}$ precipitate. Finally, the reaction between $\mathrm{Se}^{2-}$ and $\mathrm{Zn}^{2+}$ form the $\mathrm{ZnSe}$ nanostructure and $\mathrm{N}_{2} \mathrm{H}_{4} \cdot 2 \mathrm{H}_{2} \mathrm{O}$ is critical to the formation of ZnSe. Based on eqn (1) and (2), $\mathrm{N}_{2} \mathrm{H}_{4} \cdot 2 \mathrm{H}_{2} \mathrm{O}$ assists selenium dissolution and acts as a powerful reducing agent in the alkaline solution. It has been reported ${ }^{37,38}$ that $\mathrm{N}_{2} \mathrm{H}_{4} \cdot 2 \mathrm{H}_{2} \mathrm{O}$ helps to form the proper coordination molecular template in the synthesis of zinc chalcogenides. We believe that $\mathrm{N}_{2} \mathrm{H}_{4} \cdot 2 \mathrm{H}_{2} \mathrm{O}$ has the proper coordination ability with zinc to favor the growth of ZnSe nanocrystals. Addition of $\mathrm{N}_{2} \mathrm{H}_{4} \cdot 2 \mathrm{H}_{2} \mathrm{O}$ has a significant influence on the morphology of the $\mathrm{ZnSe}$ nanostructures. Experiments performed in the absence of $\mathrm{N}_{2} \mathrm{H}_{4} \cdot 2 \mathrm{H}_{2} \mathrm{O}$ reveal little dissolution of the Se powder and $\mathrm{ZnSe}$ nanostructures are not formed efficiently. By increasing the $\mathrm{N}_{2} \mathrm{H}_{4} \cdot 2 \mathrm{H}_{2} \mathrm{O}$ concentration, the nanostructures begin to grow. In fact, $\mathrm{N}_{2} \mathrm{H}_{4} \cdot 2 \mathrm{H}_{2} \mathrm{O}$ not only serves as a solvent, but also influences the surface structure of $\mathrm{ZnSe}$ in the formation process. The process is illustrated in Scheme 1.

The FE characteristics are analyzed according to the FowlerNordheim (F-N) theory as shown in the following: ${ }^{39}$

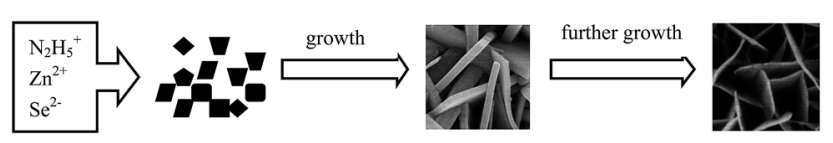

$$
\ln \left(J / E^{2}\right)=\ln \left(A \beta^{2} / \Phi\right)-\left(B \Phi^{3 / 2} / \beta E\right),
$$

where $J$ is the current density, $E$ is the applied electrical field, $\beta$ is the enhancement factor, $\Phi$ is work function, and $A$ and $B$ are constants with the value of $1.54 \times 10^{-6} \mathrm{AeV} \mathrm{V}^{-2}$ and $6.83 \times 10^{3}$ $\mathrm{eV}^{-3 / 2} \mu \mathrm{m}^{-1}$, respectively. By adopting the Wenzel, Kramers, Brillouin (WKB) approximation, eqn (5) can be simplified as follows:

$$
\ln \left(J / E^{2}\right)=\ln a-b(1 / E),
$$

where $a$ and $b$ are constants, $J$ is the field emission current density, and $E$ is the applied field. According to eqn (6), the F-N plot can be drawn and the degree of linearity suggests that it is not thermal emission but instead field emission. The $J-E$ curves and $\mathrm{F}-\mathrm{N}$ plots are presented in Fig. 6. Fig. 6(a) shows the turn-on fields defined as the electric field corresponding to a current density of $10 \mu \mathrm{A}$ $\mathrm{cm}^{-2}$ are $4.26 \mathrm{~V} \mathrm{~mm}^{-1}$ (Sample 1), $3.29 \mathrm{~V} \mathrm{\mu m}^{-1}$ (Sample 2), $2.49 \mathrm{~V}$ $\mu \mathrm{m}^{-1}$ (Sample 4), and $1.51 \mathrm{~V} \mu \mathrm{m}^{-1}$ (Sample 3). As expected, Sample 3 with thin nanowalls show the smallest turn-on field.

Fig. 6(b) shows the relationship between $\ln \left(J / E^{2}\right)$ and $1 / E$. The F-N plots show a roughly linear relationship implying that quantum tunneling is responsible for the emission. By using the slopes of F-N plot and assuming a work function of $4.84 \mathrm{eV}$ for bulk ZnSe, ${ }^{40} \beta$ was calculated to be $1109,1896,3678$, and 4797 for samples $1,2,3$, and 4 , respectively. The field enhancement factor $(\beta)$ depends on the areal density and geometry of the emitters. Comparing the four samples with different, the Sample 3 with medium density of emission spots shows higher field enhancement than the Sample 2 with high density of emission spots due to the relatively low density of emitters preventing the electrostatic screening effect. ${ }^{41}$ The Sample 3 with thin nanowalls exhibits higher emission than the Sample 1 with thick nanowalls due to the structures with thin sheets and sharp edges. Moreover, Sample 3 have sharper edgelike structures than Sample 4 with curly edges thus favoring field emission. All in all, ZnSe nanowalls composed of thin nanosheets (Sample 3) possessing low turn-on field and large enhancement factor may be ascribed to the desired thickness of nanowalls and sharp edges on the surface, as shown in Fig. 2(c). Hence, the enhanced field emission performance can be achieved by adjusting the thickness of ZnSe nanowalls.

Fig. 6(c-f) show the field emission stability of the 4 samples by plotting the emission current density as a function of time. The field-emission current density of 4 samples is monitored for $200 \mathrm{~min}$ at an initial emission current density of $3 \mathrm{~mA} \mathrm{~cm}{ }^{-2}$. Besides, the field emission current density of Sample 3 was monitored at an initial emission current density of $1.5 \mathrm{~mA} \mathrm{~cm}^{-2}$ and $5 \mathrm{~mA} \mathrm{~cm}^{-2}$. The result suggests that the overall fluctuations in field emission current of Sample 3 does not show significant degradation indicating excellent emission stability.

Table 1 lists the turn-on fields and field enhancement factors of different $\mathrm{ZnSe}$ field emitters reported in the literature and from this work. The FE performance of the ZnSe nanowalls described here are generally comparable to other ZnSe nanostructures such as nanoribbons, ${ }^{40}$ nanoarrays, ${ }^{42}$ nanoflowers ${ }^{43}$ and nanograsses. ${ }^{44}$

Scheme 1 Formation process of the ZnSe nanowalls. 

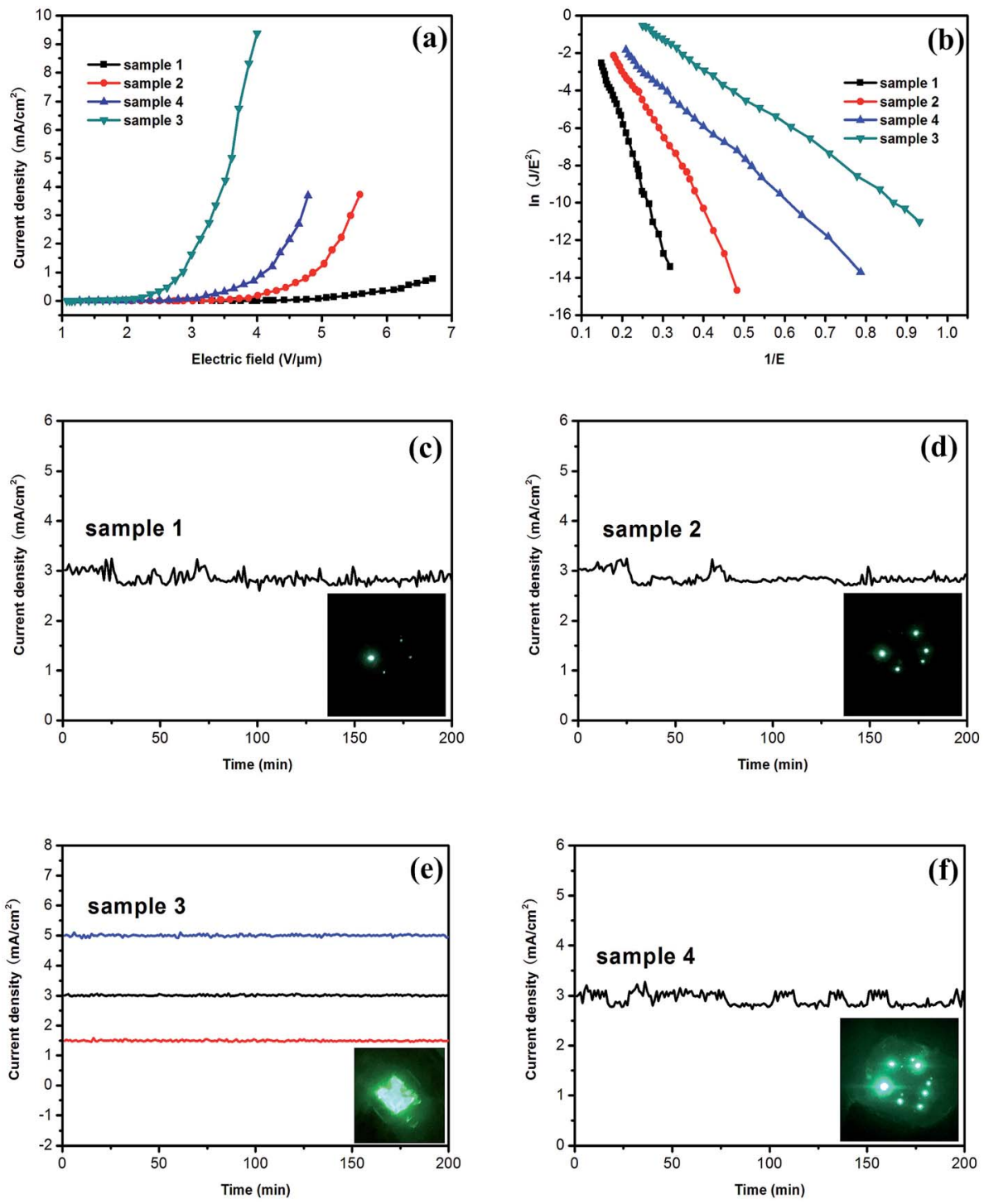

Fig. 6 (a) Field emission J-E curve, (b) FN plot, and (c-f) long-term field emission current stability test conducted on samples 1-4 (the insets show the fluorescence field emission image of the corresponding samples).

Table 1 Field emission characteristic of the ZnSe nanostructures

\begin{tabular}{lll}
\hline ZnSe nanostructures & $\begin{array}{l}\text { Turn-on field } \\
\left(\mathrm{V} \mu \mathrm{m}^{-1} \text { at } \mu \mathrm{Am}^{-2}\right)\end{array}$ & $\begin{array}{l}\text { Field enhancement } \\
\text { factor }(\beta)\end{array}$ \\
\hline ZnSe nanoribbons $^{40}$ & 5.00 at 0.1 & 1382 \\
ZnSe nanoarrays $^{42}$ & 4.05 at 0.1 & 1228 \\
ZnSe nanoflowers $^{43}$ & 3.5 at 10 & 3499 \\
ZnSe nanograsses $^{44}$ & 4.5 at 10 & 2715 \\
ZnSe nanowalls & 1.51 at 10 & 4797 \\
[this paper] & &
\end{tabular}

\section{Conclusion}

High-quality sheet-based ZnSe nanomaterials with controlled morphologies are synthesized hydrothermally. The ZnSe nanowalls are composed of radially aligned ZnSe nanosheets of singlecrystal ZnSe nanocrystals with a cubic zinc blende structure. The concentration of hydrazine hydrate plays an important role in the formation of the sheet-based ZnSe nanostructures. The investigation of field emission properties indicated that the ZnSe nanowalls composed of the thin and no curly nanosheets show a low turn-on field of $1.51 \mathrm{~V} \mathrm{\mu m}^{-1}$ and high enhancement factor of 4797 . The samples have potential in flat-panel-displays.

\section{Acknowledgements}

This work was supported by the Natural Science Foundation of China (No. 61072003) and City University of Hong Kong Applied Research Grant (ARG) No. 9667122.

\section{References}

1 A. S. Pawbake, M. S. Pawar, S. R. Jadkar and D. J. Late, Nanoscale, 2016, 8, 3008-3018. 
2 K. K. Naik, R. T. Khare, R. V. Gelamo, M. A. More, R. Thapa, D. J. Late and C. S. Rout, Mater. Res. Express, 2015, 2, 095011.

3 C. S. Rout, P. D. Joshi, R. V. Kashid, D. S. Joag, M. A. More, A. J. Simbeck, M. Washington, S. K. Nayak and D. J. Late, Sci. Rep., 2013, 3, 3282.

4 S. R. Suryawanshi, A. S. Pawbake, M. S. Pawar, S. R. Jadkar, M. A. More and D. J. Late, Mater. Res. Express, 2016, 3, 035003.

5 M. S. Pawar, P. K. Bankar, M. A. More and D. J. Late, RSC Adv., 2015, 5, 88796-88804.

6 M. B. Erande, M. S. Pawar and D. J. Late, ACS Appl. Mater. Interfaces, 2016, 8, 11548-11556.

7 L. D. Bharatula, M. B. Erande, I. S. Mulla, C. S. Rout and D. J. Late, RSC Adv., 2016, 6, 105421-105427.

8 Y. B. Zhu, C. Wang, N. Petrone, J. Yu, C. Nuckolls, J. Hone and Q. Lin, Appl. Phys. Lett., 2015, 106, 123503.

9 D. J. Late, P. A. Shaikh, R. Khare, R. V. Kashid, M. Chaudhary, M. A. More and S. B. Ogale, ACS Appl. Mater. Interfaces, 2014, 6, 15881-15888.

10 M. B. Erande, S. R. Suryawanshi, M. A. More and D. J. Late, Eur. J. Inorg. Chem., 2015, 19, 3102-3107.

11 S. R. Suryawanshi, M. A. More and D. J. Late, RSC Adv., 2016, 6, 112103-112108.

12 S. R. Suryawanshi, P. S. Kolhe, C. S. Rout, D. J. Late and M. A. More, Ultramicroscopy, 2015, 149, 51-57.

13 R. Khare, D. B. Shinde, S. Bansode, M. A. More, M. Majumder, V. K. Pillai and D. J. Late, Appl. Phys. Lett., 2015, 106, 023111.

14 S. R. Suryawanshi, M. A. More and D. J. Late, J. Vac. Sci. Technol., B: Nanotechnol. Microelectron.: Mater., Process., Meas., Phenom., 2016, 34, 041803.

15 J. Q. Wu, S. Z. Deng, N. S. Xu and J. Chen, Appl. Surf. Sci., 2014, 292, 454-461.

16 S. Jana, S. Das, N. S. Das and K. K. Chattopadhyay, Mater. Res. Bull., 2010, 45, 693-698.

17 V. C. Anitha, A. N. Banerjee, S. W. Joo and B. K. Min, Nanotechnology, 2015, 26, 355705.

18 X. P. Feng, Y. X. Li, H. B. Liu, Y. L. Li, S. Cui, N. Wang, L. Jiang, X. F. Liu and M. J. Yuan, Nanotechnology, 2007, 18, 145706.

19 Y. J. Zhang, H. Li, L. Jiang, H. B. Liu, C. Y. Shu, Y. L. Li and C. R. Wang, Appl. Phys. Lett., 2011, 98, 113118.

20 B. B. Wang, K. Ostrikov, T. van der Laan, K. Zheng, J. J. Wang, Y. P. Yan and X. J. Quan, J. Mater. Chem. C, 2013, 1, 7703-7708.

21 C. S. Rout, R. Khare, R. V. Kashid, D. S. Joag, M. A. More, N. A. Lanzillo, M. Washington, S. K. Nayak and D. J. Late, Eur. J. Inorg. Chem., 2014, 31, 5331-5336.

22 G. Kiriakidis, K. Moschovis, P. Uusimaa, A. Salokatve, M. Pessa and J. Stoemenos, Thin Solid Films, 2000, 360, 195-204.
23 W. Y. Ji, P. T. Jing, W. Xu, X. Yuan, Y. J. Wang, J. L. Zhao and A. K.-Y. Jen, Appl. Phys. Lett., 2013, 103, 053106.

24 K. M. Ip, Z. Liu, C. M. Ng and S. K. Hark, Nanotechnology, 2005, 16, 1144-1147.

25 X. Gao, G. C. Tang and X. G. Su, Biosens. Bioelectron., 2012, 36, 75-80.

26 T. T. Yao, Q. Zhao, Z. P. Qiao, F. Peng, H. J. Wang, H. Yu, C. Chi and J. Yang, Chem.-Eur. J., 2011, 17, 8663-8670.

27 Y. Z. Hao and Y. Feng, Chem. J. Chin. Univ., 2008, 29, 788792.

28 Q. Z. Zeng, S. L. Xue, S. X. Wu, K. X. Gan, L. Xu, J. W. Han, W. K. Zhou, Y. T. Shi and R. J. Zou, Mater. Sci. Semicond. Process., 2015, 31, 189-194.

29 Y. Q. Zou, H. L. Li, P. Y. Ren, J. Y. Xu, L. Ma, X. X. Wang, X. P. Fan, Z. P. Shan, X. J. Zhuang, H. Zhou, X. L. Zhu, Q. L. Zhang and A. L. Pan, Mater. Lett., 2014, 129, 118-121.

30 V. Zannier, V. Grillo and S. Rubini, J. Phys. D: Appl. Phys., 2014, 47, 394005.

31 C. Fan, Q. L. Zhang, X. L. Zhu, X. J. Zhuang and A. L. Pan, Sci. Bull., 2015, 60, 1674-1679.

32 B. Feng, J. H. Yang, J. Cao, L. L. Yang, M. Gao, M. B. Wei, H. J. Zhai, Y. F. Sun and H. Song, J. Alloys Compd., 2013, 555, 241-245.

33 J. J. Yin, G. Y. Feng and S. H. Zhou, J. Optoelectron. Adv. Mater., 2015, 9, 682-687.

34 Q. L. Bao, Y. Gan, J. Li and C. M. Li, J. Phys. Chem. C, 2008, 112, 19718-19726.

35 X. Wang, X. Chen, H. Zheng, J. Jin and Z. Zhang, Appl. Phys. A: Mater. Sci. Process., 2005, 80, 511-513.

36 H. Q. Cao, Y. J. Xiao and S. C. Zhang, Nanotechnology, 2011, 22, 015604.

37 Y. J. Dong, Q. Peng and Y. D. Li, Inorg. Chem. Commun., 2004, 7, 370-373.

38 L. L. Chai, J. Du, S. L. Xiong, H. B. Li, Y. C. Zhu and Y. T. Qian, J. Phys. Chem. C, 2007, 111, 12658-12662.

39 J. Z. Xu, R. Pan, Y. W. Chen, X. Q. Piao, M. Qian, T. Feng and Z. Sun, J. Alloys Compd., 2013, 551, 348-351.

40 L. J. Zhao, Q. Pang, Y. Cai, N. Wang, W. K. Ge, J. N. Wang and S. H. Yang, J. Phys. D: Appl. Phys., 2007, 40, 3587-3591.

41 C. X. Wu, F. S. Li, Y. G. Zhang and T. L. Guo, Carbon, 2012, 50, 3622-3626.

42 Q. Su, L. J. Li, S. Y. Li and H. P. Zhao, Micro Nano Lett., 2012, 7, 1053-1055.

43 S. L. Xue, S. X. Wu, Q. Z. Zeng, P. Xie, K. X. Gan, J. Wei, S. Y. Bu, X. N. Ye, L. Xie, R. J. Zou, C. M. Zhang and P. F. Zhu, Appl. Surf. Sci., 2016, 365, 69-75.

44 W. K. Zhou, S. L. Xue, J. W. Han and P. Xie, Mater. Lett., 2014, 134, 256-258. 